\title{
CONTRIBUCIÓN AL ESTUDIO POLÍNICO DE ESPECIES ORNAMENTALES: SOLANACEAE, CONVOLVULACEAE E HYDROPHYLLACEAE.
}

\author{
M. Mar TRIGO
}

RESUMEN. Se estudian con el M.O. y con el M.E.B. la morfología polínica de 19 especies pertenecientes a las familias Solanaceae (15), Convolvulaceae (3) e Hidrophyllaceae (1) habitualmente cultivadas como ornamentales en el sur de España.

La mayoría de los táxones estudiados presentan tipos polínicos trizonocolpados o trizonocolporados, de tamaño pequeño o mediano y superficie con elementos supratectales de escaso relieve, excepto Calonyction aculeatum House e Ipomoea mutabilis Lind. que tienen granos de polen polipantoporados, de tamaño grande a muy grande y elementos supratectales de hasta $12 \mu \mathrm{m}$ de longitud.

Palabras clave. Polen, Solanaceae, Convolvulaceae, Hydrophyllaceae.

ABSTRACT. The pollen morphology of nineteen species of Solanaceae, Convolvulaceae and Hydrophyllaceae usually cultivated in southern Spain are studied by light and scanning electron microscopy. Pollen was acetolysed by the method of Erdtman (1960) modificated by Reitsma (1969) and mounted in glycerine jelly for light microscopy. For scanning electron microscopy the pollen were coated of evaporate gold.

The taxa examined have generally 3-colpate or 3-colporate pollen grains with small or medium size and without supratectal elements, except Calonyction aculeatum House and Ipomoea mutabilis Lind. that have polipantoporate pollen grains, large or very large and supretectals elements up to $12 \mu \mathrm{m}$.

Key words. Pollen, Solanaceae, Convolvulaceae, Hydrophyllaceae.

\section{INTRODUCCIÓN}

Las especies de las familias Solanaceae, Convolvulaceae e Hydrophyllaceae se encuentran distribuidas fundamentalmente por las regiones tropicales y templadas de todo el Mundo. Muchas de estas especies poseen flores grandes y vistosas por lo que se ha difundido su cultivo como plantas ornamentales, especialmente en zonas de clima cálido y subtropical. En estas zonas se ha podido observar que sus flores son repetidamente visitadas por insectos, fundamentalmente abejas, siendo probable que el polen de las mismas aparezcan en el contenido de las mieles procedentes de colmenas próximas a núcleos urbanizados.

El presente trabajo trabajo pretende ser una continuación de otros ya publicados anteriormente (Trigo, 1989; Trigo y García, 1990; Trigo, 1991) como contribución al estudio palinológico de plantas cultivadas. 


\section{MATERIAL Y MÉTODOS}

Para el estudio polínico se ha utilizado tanto material fresco, recién recolectado e incluido en ácido acético glacial, como material seco procedente del Herbario del Departamento de Biología Vegetal de la Facultad de Ciencias de la Universidad de Málaga (MGC). En todos los casos dicho material se trató siguiendo el método acetolítico de Erdtman (1960) modificado por Reitsma (1969). El montaje para su observación con el microscopio óptico se realizó usándose como medio glicerogelatina incolora y sellándose posteriormente las preparaciones con parafina. Para su observación con el microscopio electrónico de barrido (MEB) se recubrieron las muestras con oro vaporizado en atmósfera de alto vacío, apareciendo en cada una de las microfotografías obtenidas las siguientes inscripciones, de izquierda a derecha: número de archivo de la foto, kilovoltaje al que fue sometida la muestra, microescala y distancia de trabajo (WD), expresada en milímetros.

Para calcular las dimensiones de los ejes polar $(\mathrm{P})$, ecuatorial $(\mathrm{E})$ y diámetro (D), se han efectuado 30 medidas por muestra y para los demás caracteres al menos 15 observaciones. Los valores que aparecen entre paréntesis corresponden a la media aritmética y a la desviación típica del intervalo. El grosor de la exina se ha medido en la mesocolpia, en vista polar y corte óptico meridiano, o en la mesoporia en el caso de granos de polen polipantoporados. En estos últimos, el número de poros se ha calculado por medio de la fórmula de Melville (1981).

La terminología empleada en las descripciones es, fundamentalmente, la propuesta por Erdtman (1945, 1952), Faegri \& Iversen (1975), Hideux \& Ferguson (1975), Reitsma (1970), Van Campo (1958) y Wodehouse (1935).

En el texto, las especies aparecen, dentro de cada familia, ordenadas alfabéticamente, habiendose agrupado aquéllas cuyos granos de polen presentan una morfología similar. Se incluye además el areal natural de distribución de las mismas así como las referencias que hemos encontrados sobre estudios anteriores.

\section{RESULTADOS}

\section{SOLANACEAE}

Brunfelsia calycina Benth. var. floribunda Hort. (Brasil).

Polen generalmente trizonocolpado. Isopolar, con simetría radial, apareciendo a veces tipos tetracolpados y loxocolpados. Circular en v.p. y c.o.e; de subcircular a ligeramente elíptico en v.e. y c.o.m; de suboblado a prolado-esferoidal, siendo $\mathrm{P} / \mathrm{E}=0,86-1,14(0,99 \pm$ $0,07)$. Tamaño de pequeño a mediano, con valores de $\mathrm{P}=23-29(25,57 \pm 1,62) \mu \mathrm{m}$ y de $\mathrm{E}=21,5-$ $30(25,84 \pm 1,81) \mu \mathrm{m}$. Aberturas simples, de tipo colpo, terminales, a veces algo constreñidos en la parte central, con la membrana apertural escábrida y rodeadas de un margo psilado. Exina de 1 a $1,2 \mu \mathrm{m}$ de grosor, con la nexina unas tres veces más gruesa que la sexina. Téctum completo. Infratéctum sin columelas aparentes. Superficie fosulada. (Lám. I, figs. 1-4).

Referencias: se desconocen.

Cestrum cultum Francey (América tropical).

Cestrum elegans Schlecht. (México).

Polen generalmente trizonocolporado. Isopolar, con simetría radial, aunque es frecuente la aparición en C. elegans de granos de polen hexacolporados $(3+3)$ y heteropolares. Circular lobulado en v.p. y c.o.e; elíptico en v.e. y c.o.m., de oblado-esferoidal a prolado, siendo $\mathrm{P} / \mathrm{E}=$ $1,16-1,74(1,46 \pm 0,14)$ en C. cultum y $0,95-1,47(1,24 \pm 0,14)$ en C. elegans. Tamaño mediano, con valores de $\mathrm{P}=28-38,5(34,08 \pm 2,17) \mu \mathrm{m}$ en $C$. cultum y $35-45,5(40,03 \pm 2,44) \mu \mathrm{m}$ en $C$. elegans y de $\mathrm{E}=20,5-26(23,36 \pm 1,36) \mu \mathrm{m}$ en $C$. cultum y $25,5-37,5(32,48 \pm 3,46) \mu \mathrm{m}$ en $C$. 
elegans. Aberturas compuestas; ectoaberturas de tipo colpo, terminales, a veces constreñidas en la parte central, con la membrana apertural psilada; endoaberturas de tipo poro, lalongadas y bastante difusas, especialmente en $C$. cultum. Exina de 1,5 a $2 \mu \mathrm{m}$ de grosor, con la sexina ligeramente más gruesa que la nexina, formando esta última costillas a nivel de las endoaberturas. Téctum completo. Infratéctum con columelas poco patentes. Superficie estriada, con los elementos dispuestos paralelamente al eje polar. (Lám. I, figs. 5-13).

Referencias: se desconocen.

\section{Cestrum nocturnum L. (Centroamérica).}

Polen trizonocolporado, muy rara vez bizonocolpado. Isopolar, con simetría radial. Circular lobulado en v.p. y c.o.e; elíptico en v.e. y c.o.m., de suboblado a subprolado, siendo $\mathrm{P} / \mathrm{E}=0,85-1,27(1,15 \pm 0,09)$. Tamaño mediano, con valores de $\mathrm{P}=29-33(31,2 \pm 1,06) \mu \mathrm{m}$ y de $E=23-30,5(26,94 \pm 1,84) \mu \mathrm{m}$. Aberturas compuestas; ectoaberturas de tipo colpo, terminales, con la membrana apertural granulosa; endoaberturas de tipo poro, lalongadas, elípticas, con los extremos acuminados y constreñidas en la parte central, de 17-20 x 3-4 $\mu \mathrm{m}$. Exina de 2 a $2,5 \mu \mathrm{m}$ de grosor en la mesocolpia, siendo algo más gruesa alrededor de las aberturas, con la sexina ligeramente más gruesa que la nexina, formando esta última costillas a nivel de las endoaberturas. Téctum completo, muy grueso. Infratéctum con columelas muy cortas y poco patentes. Superficie psilada, con algunas fósulas y perforaciones bastante dispersas. (Lám. II, figs. 1-7).

Referencias: se desconocen.

\section{Datura suaveolens Humb. \& Bonpl. (Brasil).}

Polen trizonocolporado. Isopolar, con simetría radial. Circular en v.p. y c.o.e; de subcircular a elíptico en v.e. y c.o.m., de suboblado a oblado-esferoidal, siendo $\mathrm{P} / \mathrm{E}=0,84-0,96$ $(0,88 \pm 0,02)$. Tamaño mediano, con valores de $P=32-41(35,26 \pm 1,93) \mu \mathrm{m}$ y de $E=35-43$ $(39,77 \pm 2,02) \mu \mathrm{m}$. Aberturas compuestas; ectoaberturas de tipo colpo, muy cortas, de 8-12 x 1-4 $\mu \mathrm{m}$, dispuestas en la zona ecuatorial; endoaberturas de tipo poro, lalongadas, formando un endocíngulo de 7 a $10 \mu \mathrm{m}$ de anchura. Exina de 3 a $4 \mu \mathrm{m}$ de grosor, con la sexina de 3 a 4 veces más gruesa que la nexina, formando esta última costillas a nivel de las endoaberturas. Téctum perforado. Infratéctum columelado. Superficie supraestriada, con los elementos de superficie equinulada y dispuestos paralelamente al eje polar. (Lám. II, figs. 8-14).

Referencias: Erdtman (1952).

Nicotiana glauca R. C. Graham (Argentina y Bolívia, ampliamente naturalizada).

Polen trizonocolporado. Isopolar, radiosimétrico. De circular a subtriangular en v.p. y c.o.m., de prolado-esferoidal a prolado, siendo $\mathrm{P} / \mathrm{E}=1,03-1,47(1,15 \pm 0,11)$. Tamaño pequeño, con valores de $P=21,5-25(22,97 \pm 0,87) \mu \mathrm{m}$ y de $E=16,5-22,5(20,05 \pm 1,29) \mu \mathrm{m}$. Aberturas compuestas; ectoaberturas de tipo colpo, terminales, con la membrana apertural granulosa; endoaberturas de tipo poro, lalongadas. Exina de 1 a 1,5 $\mu \mathrm{m}$ de grosor, con la sexina dos veces más gruesa que la nexina. Téctum perforado. Infratéctum con columelas poco patentes. Superficie rugulado-perforada en la mesocolpia, haciéndose psilada en las zonas polares. (Lám. III, figs. 1-6).

Referencias: Polo y Díez (1986, 1987b).

Petunia $x$ hybrida Viem. (Las especies parentales, P. axillaris B.S.P. y P. violacea Lindl., proceden de Sudamérica).

Polen trizonocolporado. Isopolar, con simetría radial. Circular en v.p. y c.o.e; de subcircular a elíptico en v.e. y c.o.m, de oblado-esferoidal a prolado-esferoidal, siendo $\mathrm{P} / \mathrm{E}=$ $0,92-1,11(0,97 \pm 0,04)$. Tamaño de pequeño a mediano, con valores de $\mathrm{P}=22-28(24,77 \pm 1,27)$ $\mu \mathrm{m}$ y de $\mathrm{E}=23-27,5(25,50 \pm 0,92) \mu \mathrm{m}$. Aberturas compuestas; ectoaberturas de tipo colpo, con la membrana apertural granulosa; endoaberturas de tipo poro, algo difusas al M.O. Exina de 1,5 a $2 \mu \mathrm{m}$ de grosor, con la sexina unas dos veces más gruesa que la nexina. Téctum parcial, 
infratéctum columelado. Superficie estriada, con los elementos dispuestos paralelamente al eje polar. (Lám. III, figs. 7-11).

Referencias: se desconocen.

Solandra grandiflora SW. (México).

Solandra guttata Don. (México).

Polen trizonocolporado. Isopolar, con simetría radial. Circular en v.p. y c.o.e; de circular a ligeramente elíptico en v.e. y c.o.m., de suboblado a prolado-esferoidal, siendo $\mathrm{P} /$ $\mathrm{E}=0,88-1,04(0,92 \pm 0,04)$ en $S$. grandiflora y $0,85-0,96(0,92 \pm 0,03)$ en $S$. guttata. Tamaño pequeño, con valores de $\mathbf{P}=17-19(18,72 \pm 0,59) \mu \mathrm{m}$ en $S$. grandiflora y $18-21(19,61 \pm 0,65)$ $\mu \mathrm{m}$ en $S$. guttata y de E= 19-21 $(20,26 \pm 0,65) \mu \mathrm{m}$ en $S$. grandiflora y $19-23(21,15 \pm 0,80) \mu \mathrm{m}$ en $S$. guttata. Aberturas compuestas; ectoaberturas de tipo colpo, subterminales, de 9 a $11 \mu \mathrm{m}$ de longitud, dispuestos en la zona ecuatorial, con la membrana apertural granulosa; endoaberturas de tipo poro, lalongadas, de 1-1,5 x 3-4 $\mu \mathrm{m}$. Exina de aproximadamente 1,5 $\mu \mathrm{m}$ de grosor, con la sexina de 1,5 a 2 veces más gruesa que la nexina. Téctum parcial. Infratéctum columelado. Superficie retculada en las zonas polares y supraestriada en el ecuador, en donde el téctum es reticulado. (Lám. III, figs. 12-16).

Referencias: se desconocen.

Solanum jasminoides Paxt. (Brasil).

Polen trizonocolporado. Isopolar, con simetría radial. Subtriangular, con los lados convexos, en v. p. y c.o.e., angulaperturado; de circular a ligeramente elíptico en v.e. y c.o.m., de suboblado a esferoidal, siendo $\mathrm{P} / \mathrm{E}=0,78-1(0,91 \pm 0,06)$. Tamaño pequeño, con valores de $\mathrm{P}=14,5-18,5(16,41 \pm 1,09) \mu \mathrm{m}$ y de $\mathrm{E}=16-22,5(18,06 \pm 1,38) \mu \mathrm{m}$. Aberturas compuestas; ectoaberturas de tipo colpo, terminales, con la membrana apertural densamente granulosa; endoaberturas de tipo poro, lalongadas, elípticas, con los extremos agudos, de 7-13 x 2-3 $\mu \mathrm{m}$. Exina de aproximadamente $1,5 \mu \mathrm{m}$ de grosor en la mesocolpia, algo más gruesa en las zonas aperturales, con la sexina ligeramente más gruesa que la nexina, formando esta última costillas a nivel de las endoaberturas. Téctum completo. Infratéctum columelado, con columelas poco patentes. Superficie granulosa, a veces surcada por finas hendiduras del téctum a modo de ínsulas. (Lám. IV, figs. 1-6).

Referencias: se desconocen.

Cyphomandra betacea Sendt. (Sudamérica).

Solanum capsicastrum Link. (Brasil).

Solanum rantonetii Carr. (Argentina y Paraguay).

Solanum wendlandii Hook. (Costa Rica).

Polen trizonocolporado. Isopolar, con simetría radial. De circular lobulado a triangular angulaperturado en v.p. y c.o.e; de circular a elíptico en v.e. y c.o.m., de suboblado a proladoesferoidal. Tamaño pequeño (Tabla 1).

\begin{tabular}{llll}
\hline & \multicolumn{1}{c}{$\mathbf{P}$} & \multicolumn{1}{c}{$\mathbf{E}$} & \multicolumn{1}{c}{$\mathbf{P} / \mathbf{E}$} \\
\hline C. betacea & \multicolumn{1}{c}{ 16,5-19(18,34 $\pm 0,66) \mu \mathrm{m}$} & $16,5-20(18,50 \pm 0,75) \mu \mathrm{m}$ & $0,91-1,04(0,99 \pm 0,03)$ \\
S. capsicastrum & $13,5-17(15,2 \pm 0,73) \mu \mathrm{m}$ & $13-16(14,42 \pm 0,71) \mu \mathrm{m}$ & $0,94-1,12(1,05 \pm 0,05)$ \\
S. rantonetii & $14,5-16,5(15,64 \pm 0,48) \mu \mathrm{m}$ & $17,5-19(18,41 \pm 0,49) \mu \mathrm{m}$ & $0,82-0,89(0,85 \pm 0,02)$ \\
S. wendlandii & $14,5-17(15,90 \pm 0,63) \mu \mathrm{m}$ & $15-18,5(17,29 \pm 0,73) \mu \mathrm{m}$ & $0,82-1,02(0,92 \pm 0,05)$ \\
\hline
\end{tabular}

Tabla 1 .

Aberturas compuestas; ectoaberturas de tipo colpo, terminales, a menudo constreñidas en la zona ecuatorial, donde la exina se engruesa considerablemente sin que a veces parezca existir discontinuidad de la ectexina; endoaberturas de tipo poro, lalongadas, largamente elípticas, con los extremos acuminados y constreñidas en la parte central; membrana apertural 
granulosa. Exina de 1,2 a 1,6 um de grosor, con la sexina ligeramente más gruesa que la nexina, formando esta última costillas a nivel de las endoaberturas. Téctum completo. Infratéctum con columelas cortas y poco patentes. Superficie densa y regularmente equinulada, con espínulas menores de 0,4 $\mu \mathrm{m}$ de altura. (Lám. IV, figs. 7-12 y Lám. V, figs. 1-6).

Referencias: se desconocen.

Streptosolen jamesonii Miers. (Centroamérica, Colombia y Ecuador).

Polen 7-zonocolporado, rara vez 6-zonocolporado. Isopolar, con simetría radial. De circular a poligonal en v.p. y c.o.e; elíptico en v.e. y c.o.m., de oblado a suboblado, siendo P/ $\mathrm{E}=0,65-0,84(0,76 \pm 0,04)$. Tamaño mediano, con valores de $\mathrm{P}=21-27(24,01 \pm 1,13) \mu \mathrm{m} y$ de $E=29-36(31,61 \pm 1,56) \mu \mathrm{m}$. Aberturas compuestas; ectoaberturas de tipo colpo, subterminales, de 10 a $13 \mu \mathrm{m}$ de longitud, con la membrana apertural rugosa; endoaberturas de tipo poro, lalongadas, formando un endocíngulo de 7 a $10 \mu \mathrm{m}$ de anchura. Exina de 1 a $1,3 \mu \mathrm{m}$ de grosor, con la sexina dos veces más gruesa que la nexina. Téctum completo. Infratéctum con columelas poco patentes. Superficie estriado-rugulada, con los elementos irregularmente dispuestos. (Lám. V, figs. 7-12).

Referencias: se desconocen.

\section{CONVOLVULACEAE}

Calonyction aculeatum House (América tropical).

Polen polipantoporado. Apolar, radiosimétrico. Circular en corte óptico, esferoidal. Tamaño muy grande siendo $\mathrm{D}=122-134(127,20 \pm 2,81) \mu \mathrm{m}$. Aberturas simples de tipo poro, circulares, de 6 a $9 \mu$ m de diámetro, regularmente dispuestas, en número de 98 a 200, por toda la superficie del grano de polen; membrana apertural granulosa. Exina de 8 a $9 \mu \mathrm{m}$ de grosor, con la nexina igual o ligeramente más gruesa que la sexina. Téctum parcial. Infratéctum columelado, con columelas largas y delgadas. Superficie reticulada, con lúmenes irregulares de aproximadamente $1 \mu \mathrm{m}$ de diámetro. Sobre los muros, como elementos supratectales aparecen gemas de muy diverso tamaño, siendo las más grandes de 6 a $8 \mu \mathrm{m}$ de altura, abruptamente constreñidas en la base. (Lám. VI, figs. 1-4).

Referencias: Erdtman (1952), Huang (1970).

Convolvulus tricolor L. (sur de Europa, norte de Africa).

Polen trizonocolpado. Isopolar, con simetría radial. Circular en v.p. y c.o.e; de circular a elíptico en v.e. y c.o.m., de suboblado a prolado, siendo $\mathrm{P} / \mathrm{E}=0,82-1,37(1,03 \pm 0,18)$. Tamaño mediano, con valores de $\mathrm{P}=36-46,5(40,16 \pm 2,78) \mu \mathrm{m}$ y de $\mathrm{E}=30,5-46(39,44 \pm$ $4,60) \mu \mathrm{m}$. Aberturas simples de tipo colpo, terminales; membrana apertural con gemas de superficie espinulosa. Exina de 3 a $3,5 \mu \mathrm{m}$ de grosor, con la sexina de 3 a 4 veces más gruesa que la nexina. Téctum perforado. Infratéctum con columelas ramificadas en la parte distal. Superficie granulosa, con perforaciones redondeadas. (Lám. VII, figs. 1-7).

Referencias: Polo y Díez (1985, 1987a).

Ipomoea mutabilis Lind. (América tropical).

Polen polipantoporado. Apolar, radiosimétrico. Circular en corte óptico, esferoidal. Tamaño grande, siendo $D=76-98(87,6 \pm 6,72) \mu \mathrm{m}$. Aberturas simples, de tipo poro, circulares, de 2 a $4 \mu \mathrm{m}$ de diámetro, regularmente dispuestas en número de 85 a 170 por toda la superficie del grano de polen; membrana apertural equinulada. Exina de 8 a $12 \mu \mathrm{m}$ de grosor, con la nexina igual o ligeramente más gruesa que la sexina. Téctum parcial. Infratéctum columelado, con columelas largas y delgadas. Superficie reticulada, con lúmenes irregulares de hasta $1,5 \mu \mathrm{m}$ de longitud y muros lisos. Como elementos supratectales aparecen espinas de 8 a $12 \mu \mathrm{m}$ de longitud ensanchadas en su mitad inferior. (Lám. VI, figs. 5-9).

Referencias: Erdtman (1952). 


\section{HYDROPHYLLACEAE}

Wigandia caracassana H. B. K. (México, Venezuela y Colombia).

Polen trizonocolporado. Isopolar, radiosimétrico. Circular en v.p. y c.o.e; de circular a elíptico en v.e. y c.o.m; de suboblado a prolado-esferoidal, siendo $\mathrm{P} / \mathrm{E}=0,83-1,02(0,91 \pm$ $0,05)$. Tamaño pequeño, con valores de $\mathrm{P}=11-14,5(12,88 \pm 0,93) \mu \mathrm{m}$ y de $\mathrm{E}=12,5-16(14,11$ $\pm 0,79) \mu \mathrm{m}$. Aberturas compuestas; ectoaberturas de tipo colpo, terminales, con la membrana apertural escábrida; endoaberturas de tipo colpo, más o menos circulares. Exina de 1,5 $\mu \mathrm{m}$ de grosor, con la sexina 1,5 veces más gruesa que la nexina, formando esta última costillas a nivel de las endoaberturas. Téctum parcial. Infratéctum columelado. Superficie reticulada, con lúmenes irregulares de hasta $1,5 \mu \mathrm{m}$ en la mesocolpia, disminuyendo de tamaño hacia las proximidades de los colpos, en donde la superficie se hace perforada; muros psilados. (Lám. VII, figs. 8-13).

Referencias: Erdtman (1952).

\section{DISCUSIÓN}

Aunque son escasas las referencias que tenemos sobre las especies aquí incluidas, los resultados obtenidos no difieren sustancialmente de la bibliografía consultada, no habiéndose encontrado diferencias significativas en lo que a tamaño se refiere al comparar los pólenes de las especies estudiadas con los de poblaciones autóctonas, a pesar de que algunos autores (Pla Dalmau, 1961; Saenz, 1978) habian recogido el hecho de que las plantas cultivadas poseen granos de polen de mayor tamaño que las poblaciones silvestres correspondientes.

Por otra parte, en algunas especies de Solanacea (Cyphomandra betacea, Solanum capsicastrum y $S$. wendlandii) se ha observado un sistema apertural muy similar al descrito por Fernández et al. (1990) para Lycopersicon sculentum donde, al parecer, no existe discontinuidad de la ectexina en la zona ecuatorial de los colpos.

\section{Anexo 1. Material examinado.}

Brunfelsia calycina: Málaga, El Parque. 5-2-85 (MGC 17136).

Calonyction aculeatum: Málaga, Camino de San Rafael. 2-9-85 (MGC 16912). Málaga, Portada Alta. 30-7-92 (MGC Cestrum cultum: Málaga, Avda. de Andalucía. 28-1-85 (MGC 17139).

Cestrum elegans: Málaga, El Retiro. 26-2-85 (MGC 17350). Málaga, Torre del Mar. 5-4-86 (MGC 17351).

Cestrum nocturnum: Málaga, Urbanización El Mayorazgo. 8-7-85 (MGC 17137).

Convolvulus tricolor: Málaga, Avda. de Andalucía. 27-5-86 (MGC 16906).

Cyphomandra betacea: Málaga, Torre del Mar, Paseo Marítimo. 13-4-85 (MGC 16576).

Datura suaveolens: Málaga, Jardín de la Facultad de Medicina. 12-2-92 (MGC 17452).

Ipomoea mutabilis: Málaga, Urbanización El Limonar. 2-6-85 (MGC 16914). Málaga, Ciudad Jardín. 15-5-92 (MGC 17453).

Nicotiana glauca: Málaga, Plaza de Babel. 30-7-92 (MGC 17454).

Petunia x hybrida: Málaga, Jardines de Pedro Luis Alonso. 13-5-85 (MGC 17142).

Solandra grandiflora: Málaga, Urbanización El Limonar. 23-1-85 (MGC 17143).

Solandra guttata: Málaga, Pedregalejo. 17-2-85 (MGC 17144). Málaga, El Candado. 8-3-86. (MGC 16571).

Solanum capsicastrum: Málaga, Carranque. 29-4-85 (MGC 17145).

Solanum jasminoides: Málaga, Urbanización El Limonar. 10-2-85 (MGC 17146).

Solanum rantonnetii: Málaga, Paseo de Reding. 29-9-85 (MGC 17147).

Solanum wendlandii: Málaga, Urb. Cerrado de Calderón. 24-10-91 (MGC 17464).

Streptosolen jamesonii: Málaga, Nerja, jardines de La Cueva. 12-4-86 (MGC 16645).

Wigandia caracassana: Málaga, Urbanización El Limonar. 28-1-85 (MGC 16610). 


\section{BIBLIOGRAFÍA}

ERDTMAN, G. -1945- Polen morphology and plant taxonomy. III. Morina L. with addition on morphological terminology. Svensk. Bot. Tidskr., 39: 187-191.

ERDTMAN, G. -1952-Pollen morphology and plant taxonomy. Angiosperms (An introducction to palynology). Hafner Publ. Co., Stockholm.

ERDTMAN, G. - 1960- The acetolysis method. Svensk. Bot. Tidskr., 54: 561-564.

FAEGRI, K. \& J. IVERSEN -1975- Texbook of pollen analysis. Munksgaard, Copenhagen.

FERNANDEZ, M.C., J.D. ALCHE y M.I. RODRIGUEZ-GARCIA -1990- Ultraestructura de la pared del grano de polen de Lycopersicum esculentum. En G. BLANCA, C. DIAZ DE LA GUARDIA, M. C. FERNANDEZ, M. GARRIDO, M. I. RODRIGUEZ-GARCIA y A. T. ROMERO GARCIA. Polen, esporas y sus aplicaciones. Servicio de reprografía de la Facultad de Ciencias de la Universidad de Granada.

HIDEUX, M. \& I. K. FERGUSON -1975- Stereostructure de l'exine des Saxifragales: proposition d'une description logique et schematique. Bull. Soc. Bot. France, 122: 57-67.

HUANG, T. C. -1970- Pollen grains of formosan plants (6). Taiwania, 15: 13-179.

LEWIS, W. H., P. VINAY \& V. E. ZENGER - 1983-Airborne and allergenic pollen of North America. The Johns Hopkins University Press, Baltimore.

MELVILLE, R. - 1981- Surface tension, diffusion and the evolution and the morphogenesis of pollen aperture patterns. Pollen and spores, 23: 179-203.

PLA DALMAU, J. M. -1961- Polen. Talleres gráficos D.C.P., Gerona.

POLO, J.M. y M. J. DIEZ - 1985- Contribución al atlas palinológico de Andalucía Occidental II. Convolvulaceae (s.I.). Lagascalia, 13: 239-254.

POLO, J.M. y M. J. DIEZ - 1986-Contribución al atlas palinológico de Andalucía Occidental IV. Solanaceae. Lagascalia, 14: 45-66.

POLO, J. M. y M. J. DIEZ - 1987a- Convolvulaceae. En B. VALDÉS, M. J. DÍEZ e I. FERNÁNDEZ (eds.). Atlas polínico de Andalucía Occidental. Instituto de Desarrollo Regional de la Universidad de Sevilla y Excma. Diputación de Cádiz, Sevilla.

POLO, J. M. y M. J. DIEZ - 1987b- Solanaceae. En B. VALDÉS, M. J. DÍEZ E I. FERNÁNDEZ (eds.). Atlas polínico de Andalucía Occidental. Instituto de Desarrollo Regional de la Universidad de Sevilla y Excma. Diputación de Cádiz, Sevilla.

REITSMA, T. - 1969- Size modification of recent pollen grains under different treatments. Rev. Paeobot. Palynol., 9: 175-202.

REITSMA, T. - 1970- Suggestions towards unification of descriptive terminology of Angiosperms pollen grains. Rev. Paleobot. Palinology, 10: 39-60.

SAENZ, C. -1978- Polen y esporas. Editorial Blume, Madrid.

TRIGO, M. M. - 1989- Aportación al estudio palinológico de la flora ornamental de la ciudad de Málaga: Gimnospermas. Acta Bot. Malacitana, 14: 238-244.

TRIGO, M. M.e I.GARCIA - 1990-Morfología polínica de plantas ornamentale : Leguminosas. Acta Bot. Malacitana, 15: 45-67.

TRIGO, M. M. - 1991- Contribución al estudio polínico de especies ornamentales: Bignoniaceae. Acta Bot. Malacitana, 16 (2): 455-466.

VAN CAMPO, M. -1958-Palinologie africaine II. Bull. Inst. Franç. Afrique Noire, sér. A, Sci. Nat., 20: 753-760.

WODEHOUSE, R. P. -1935- Pollen grains. McGraw-Hill, New York. U.S.A.

(Aceptado para su publicación en Junio de 1992)

Dirección de la autora. Departamento de Biología Vegetal. Facultad de Ciencias. Universidad de Málaga. Apdo. 59. 29080-Málaga. 

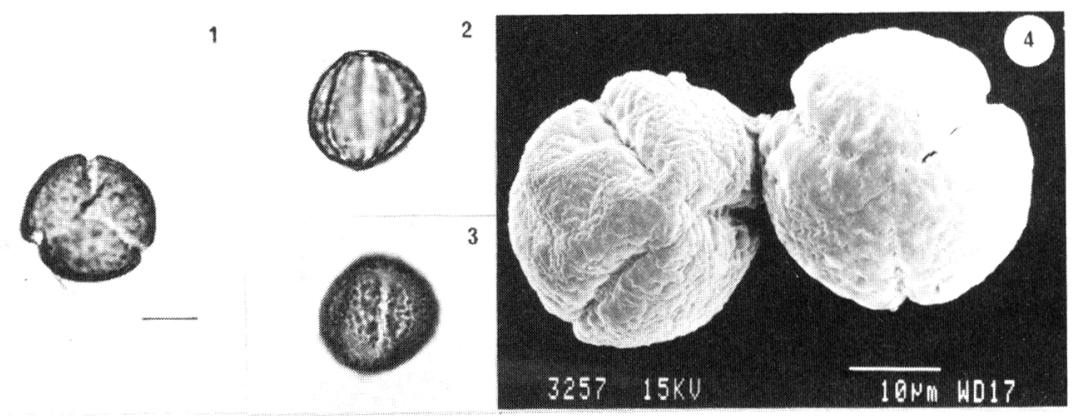

5

6

7

8

9
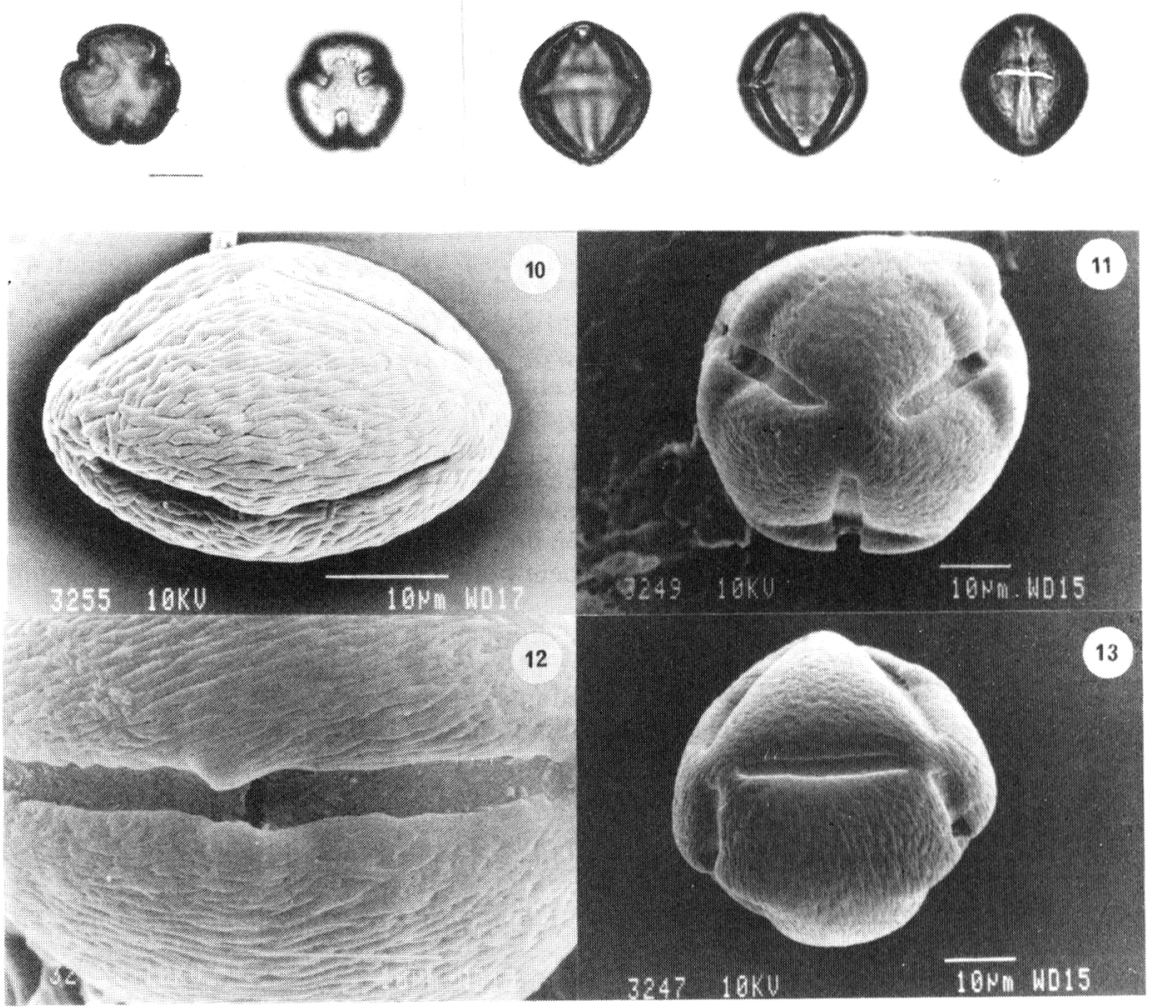

Lámina I. Brunfelsia calycina: 1) v.p. y c.o.e. 2) v.e. y c.o.m. 3) v.e., abertura y superficie. 4) Aspecto general del polen. Cestrum cultum: 5) v.p. y c.o.e 6) v.p., superficie. 7) v.e. y c.o.m. 8) v.e., costillas. 9) v.e., sistema apertural. 10) Aspecto general del polen, v.e. Cestrum elegans: 11) Aspecto general del polen, v.p. 12) Detalle de la ornamentación y el sistema apertural. 13)

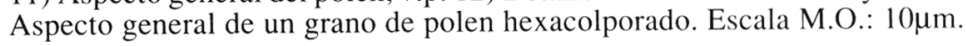




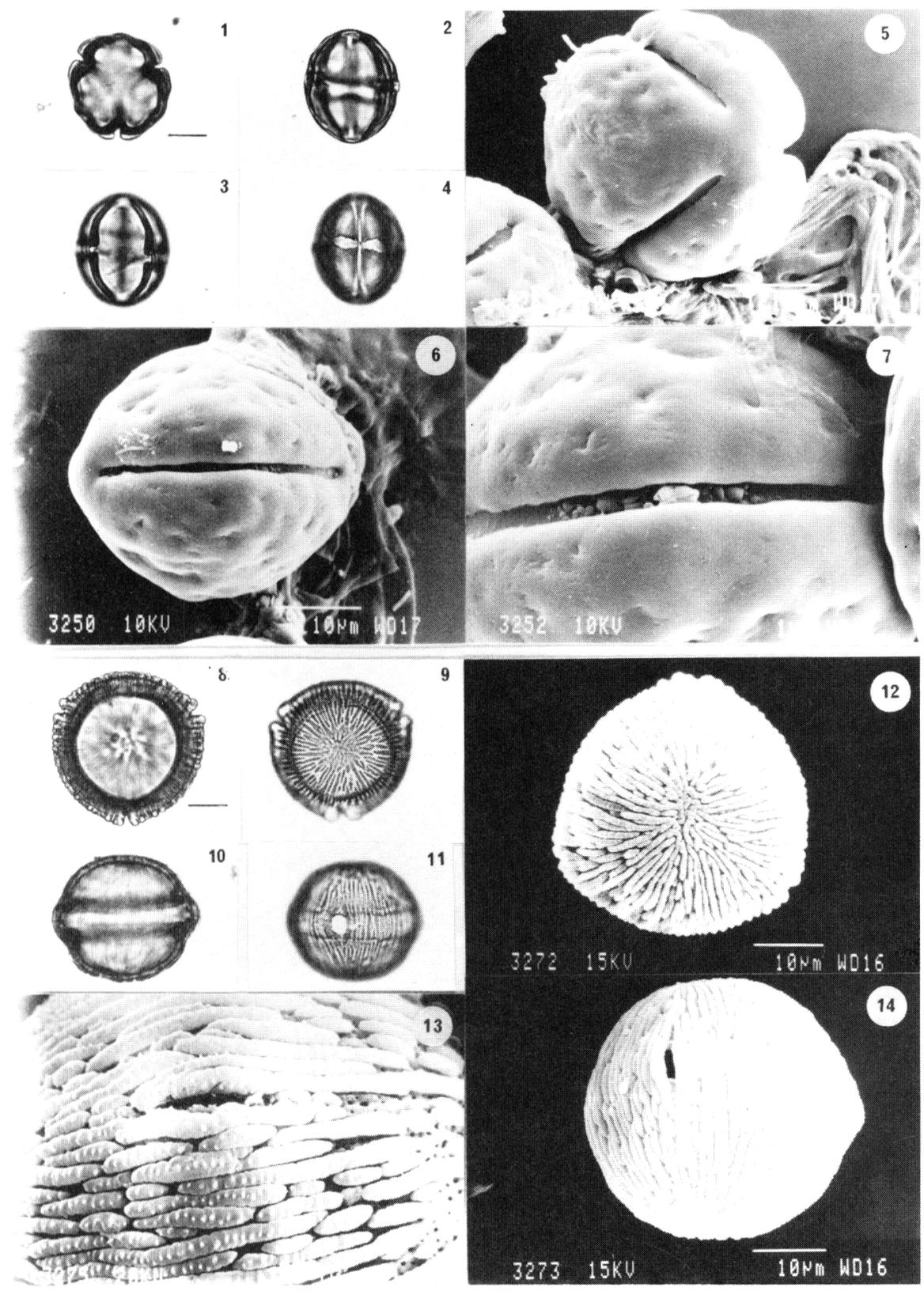

Lámina II. Cestrum nocturnum: 1) v.p. y c.o.e. 2) v.e. y c.o.m. 3) v.e., costillas. 4) v.e., sistema apertural. 5) Aspecto general del polen, v.p. 6) Aspecto general del polen, v.e. 7) Detalle de la superficie y abertura. Datura suaveolens: 8) v.p. y c.o.e. 9) v.p., superficie. 10) v.e. y c.o.m. 11) v.e., sistema apertural y superficie. 12) Aspecto general del polen, v.p. 13) Detalle de la superficie y abertura. 14) Aspecto general del polen, v.e. Escala M.O.: 10um. 

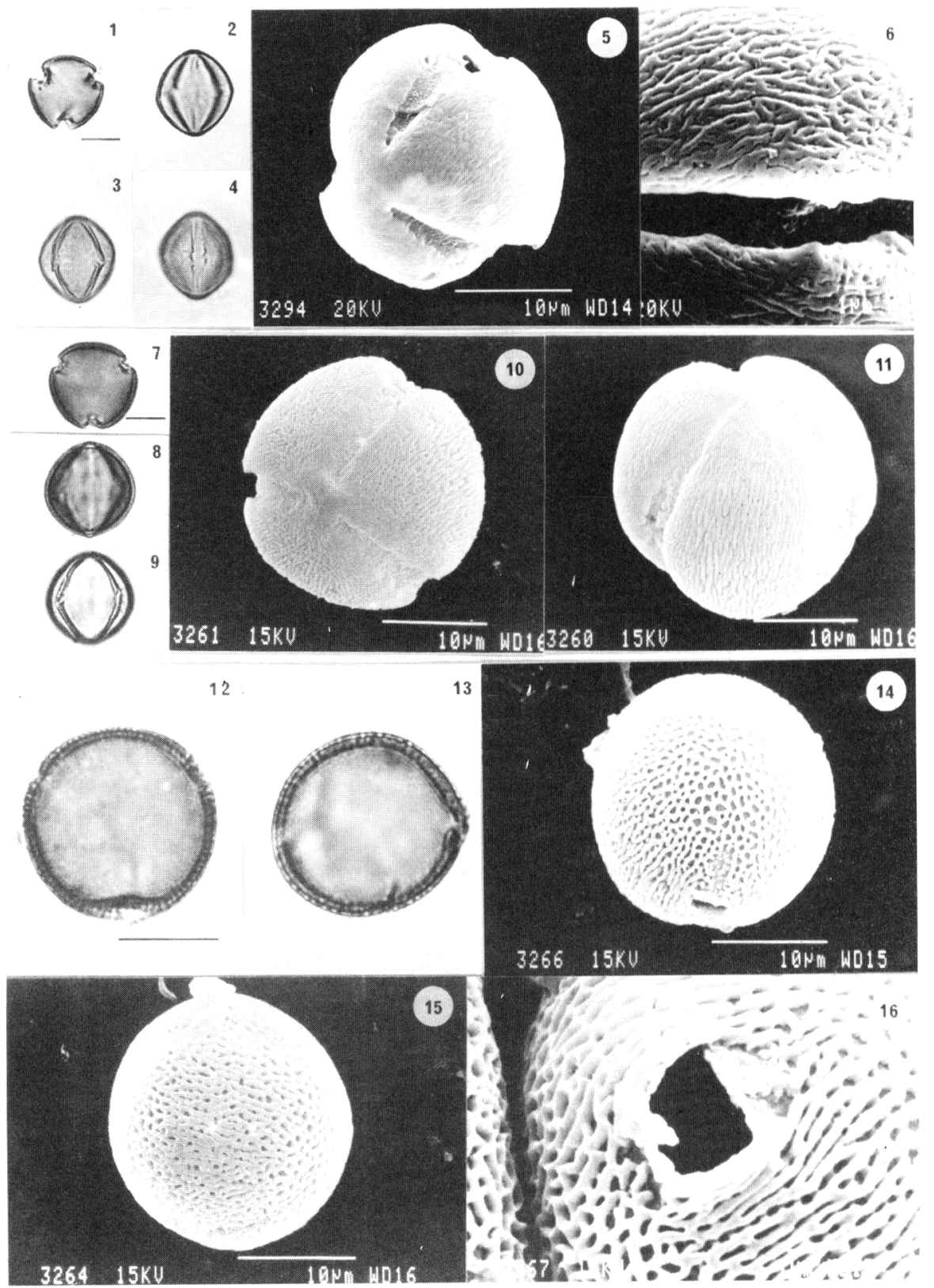

Lámina III. Nicotiana glauca: 1) v.p. y c.o.e. 2) v.e. y c.o.m. 3) v.e., costillas 4) v.e., sistema apertural 5) Aspecto general del polen. 6) Detalle de la superficie y abertura. Petunia r hybrida: 7) v.p. y c.o.e. 8) v.e. y c.o.m. 9) v.e., costillas 10) Aspecto general del polen. v.p. 11) Aspecto general del polen, v.e. Solandra grandiflora: 12) v.p. y c.o.e. 13) v.e. y c.o.m. 15) Aspecto general del polen, v.e. Solandra guttata: 14) Aspecto general del polen, v.p. 16) Detalle de la ornamentación y abertura. Escala M.O.: $10 \mu \mathrm{m}$. 

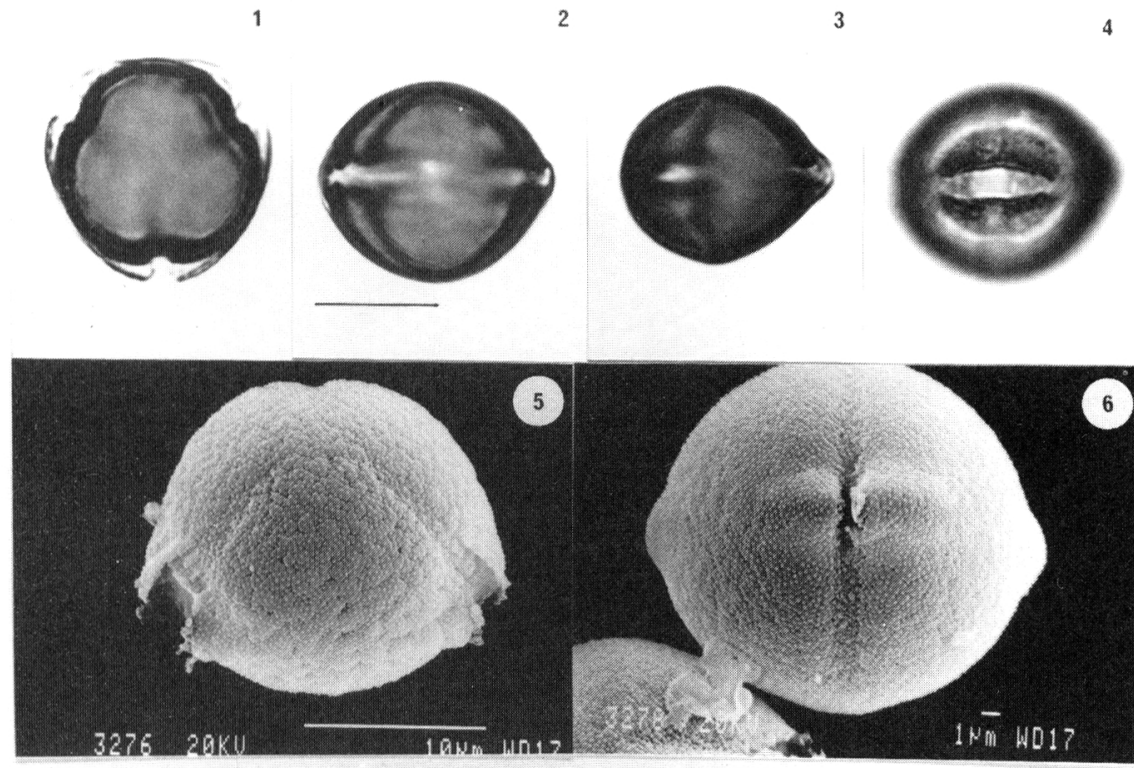

7.

8
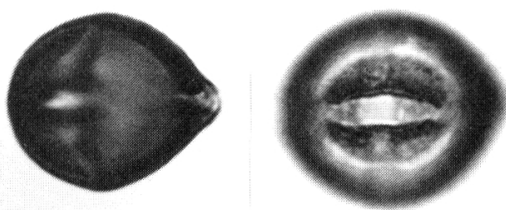

10
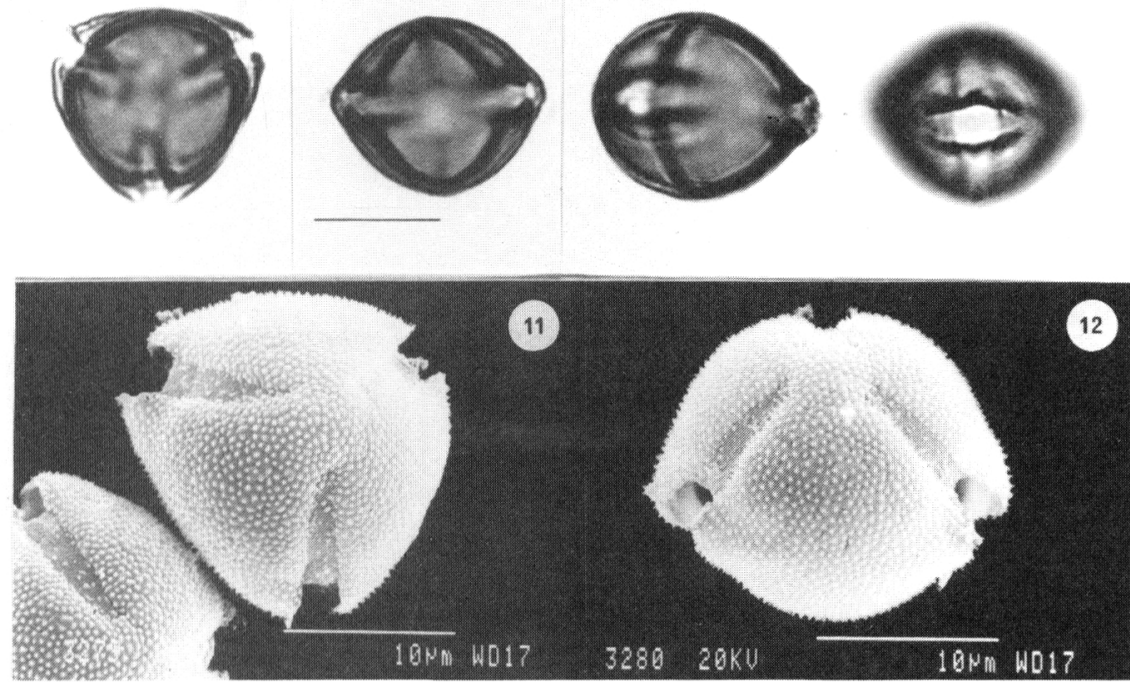

Lám. IV. Solanum jasminoides: 1) v.p. y c.o.e. 2) v.e. y c.o.m. 3) v.e., costillas 4) v.e., sistema apertural 5) Aspecto general del polen, v.p. 6) Aspecto general del polen, v.e. Solanum rantonetii: 7) v.p. y c.o.e. 8) v.e. y c.o.m. 9) v.e., costillas 10) v.e., sistema apertural 11) Aspecto general del polen, v.p. 12) Aspecto general del polen, v.e. Escala M.O.: 10um. 

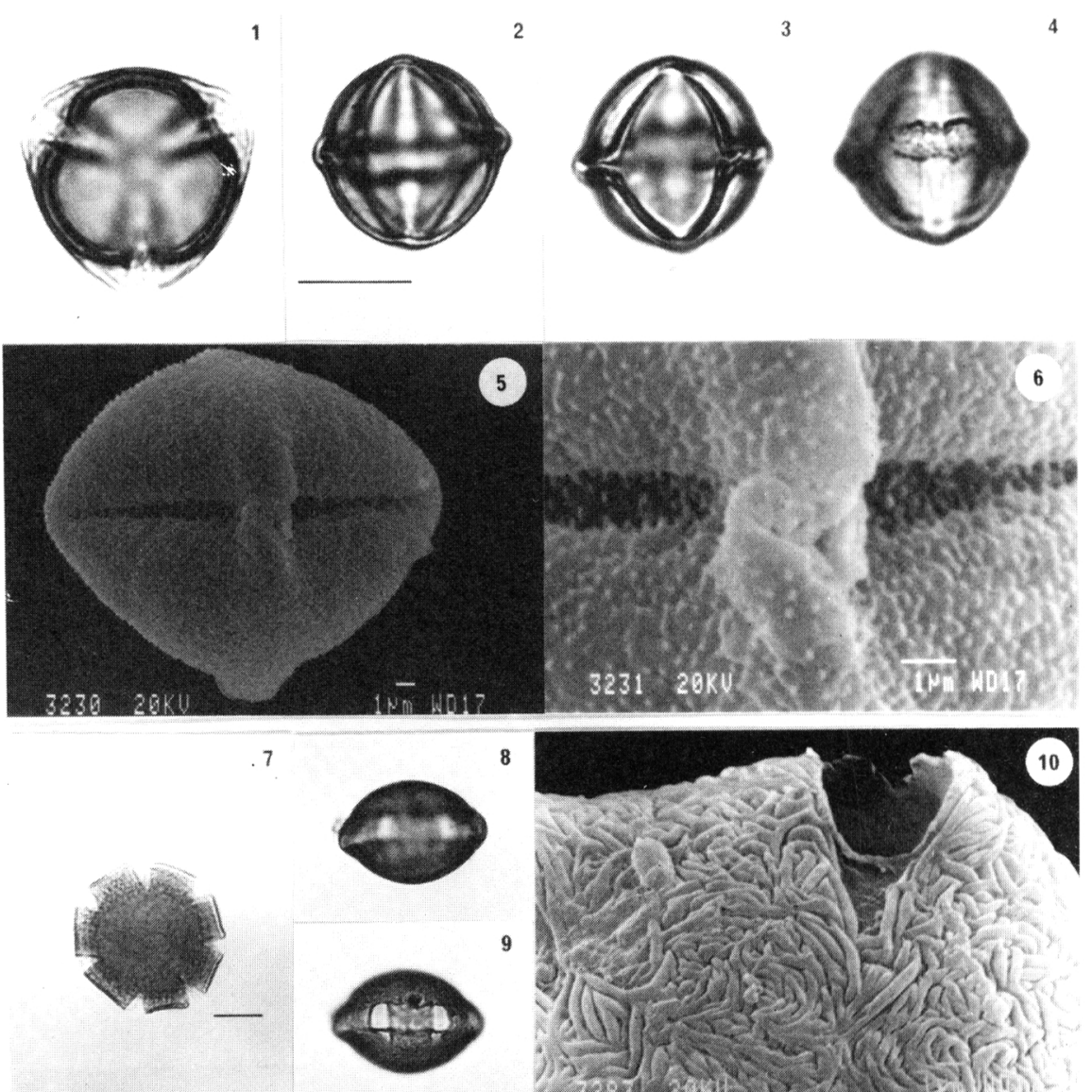

9
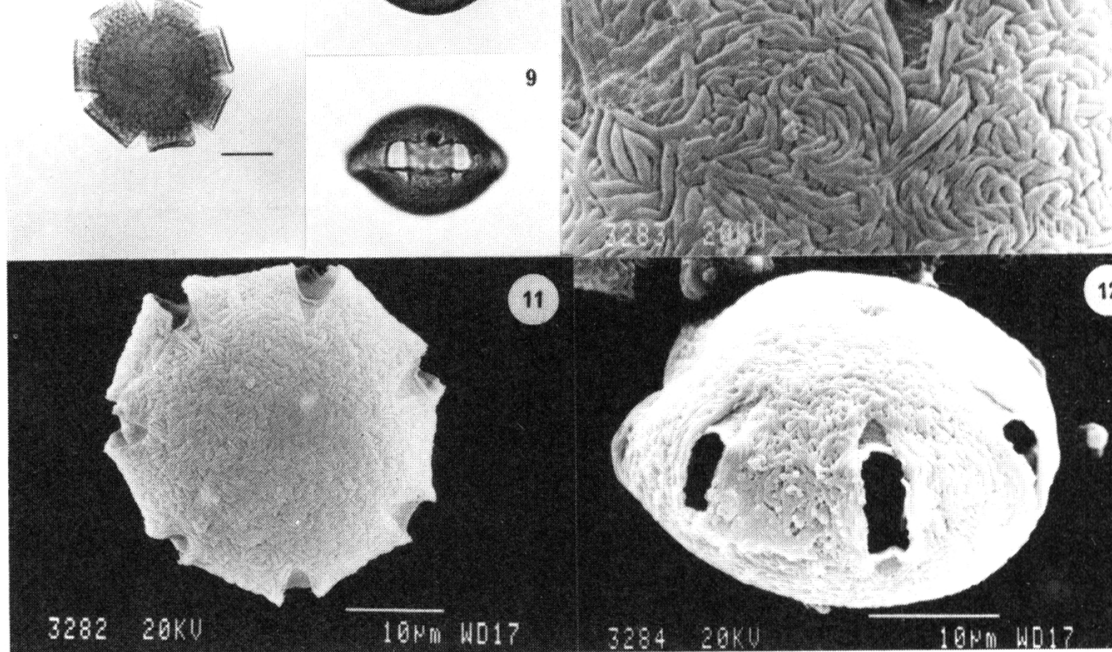

(11)
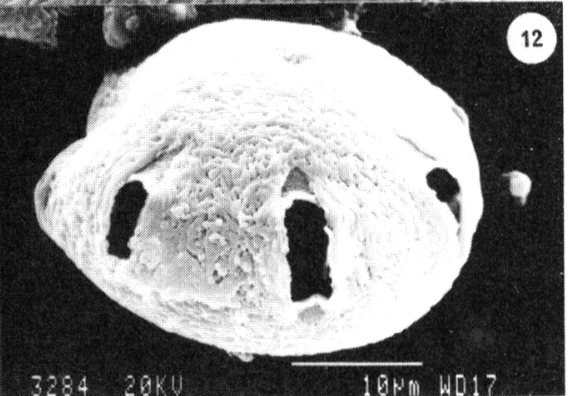

Lámina V. Cyphomandra betacea: 1) v.p. y c.o.e. 2) v.e. y c.o.m. 3) v.e., costillas 4) v.e. sistema apertural 5) Aspecto general del polen, v.e. 6) Detalle de la superficie y abertura. Streptosolen jamesonii: 7) v.p. y c.o.e. 8) v.e. y c.o.m. 9) v.e., sistema apertural 10) Detalle de la superficie y abertura. 11) Aspecto general del polen, v.p. 12) Aspecto general del polen, v.e. Escala M.O.: $10 \mu \mathrm{m}$. 


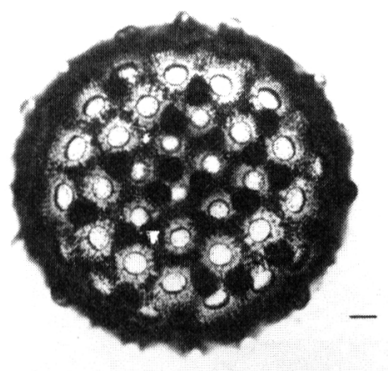

1
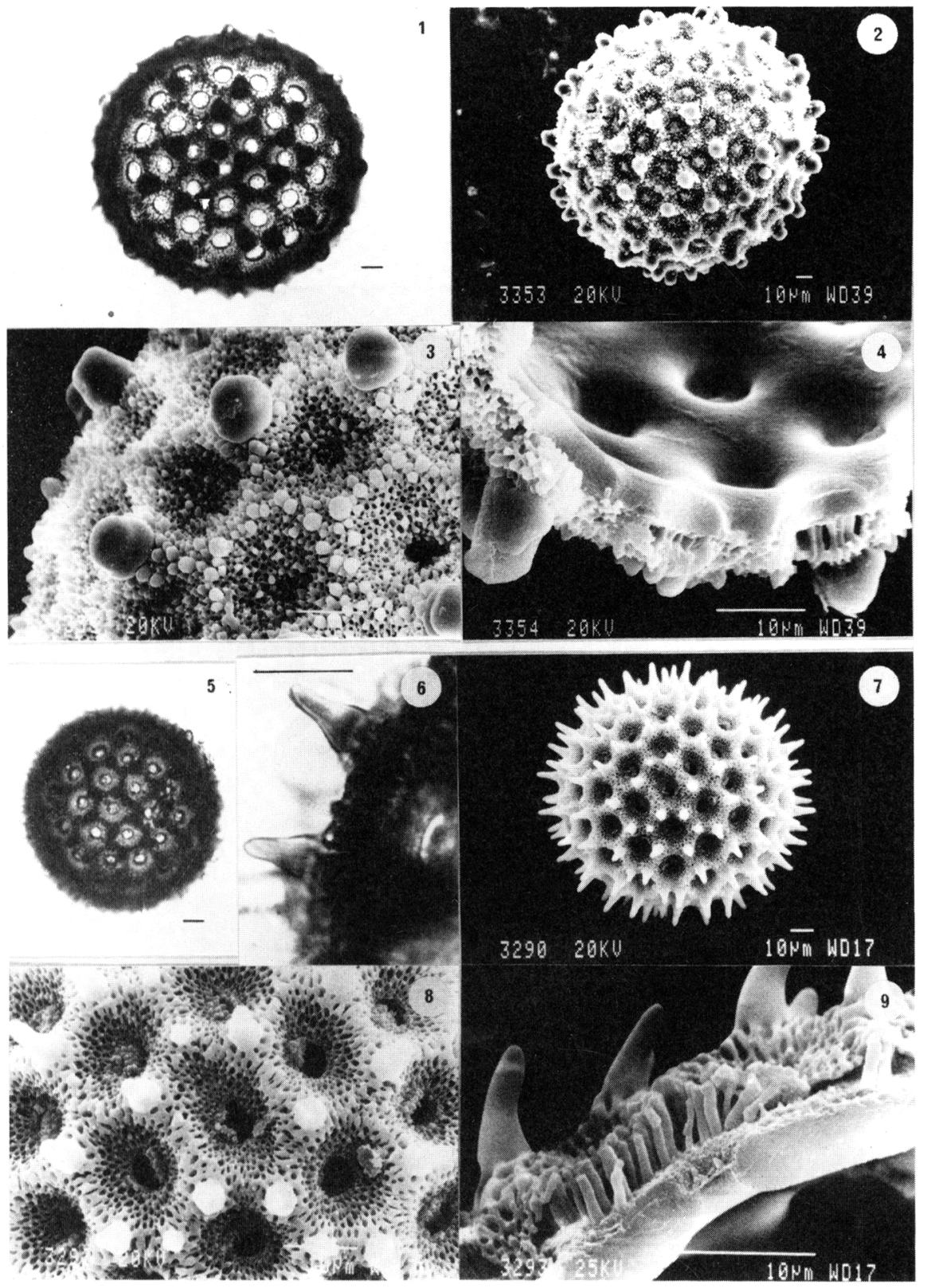

Lámina VI. Calonyction aculeatum: 1) corte óptico 2) Aspecto general del polen. 3) Detalle de la ornamentación y aberturas.4) Detalle de la estratificación de la exina. Ipomoea mutabilis: 5) corte óptico 6) Detalle de la exina y espinas 7) Aspecto general del polen. 8) Detalle de la ornamentación y aberturas. 9) Detalle de la estratificación de la exina y elementos esculturales. Escala M.O.: $10 \mu \mathrm{m}$. 


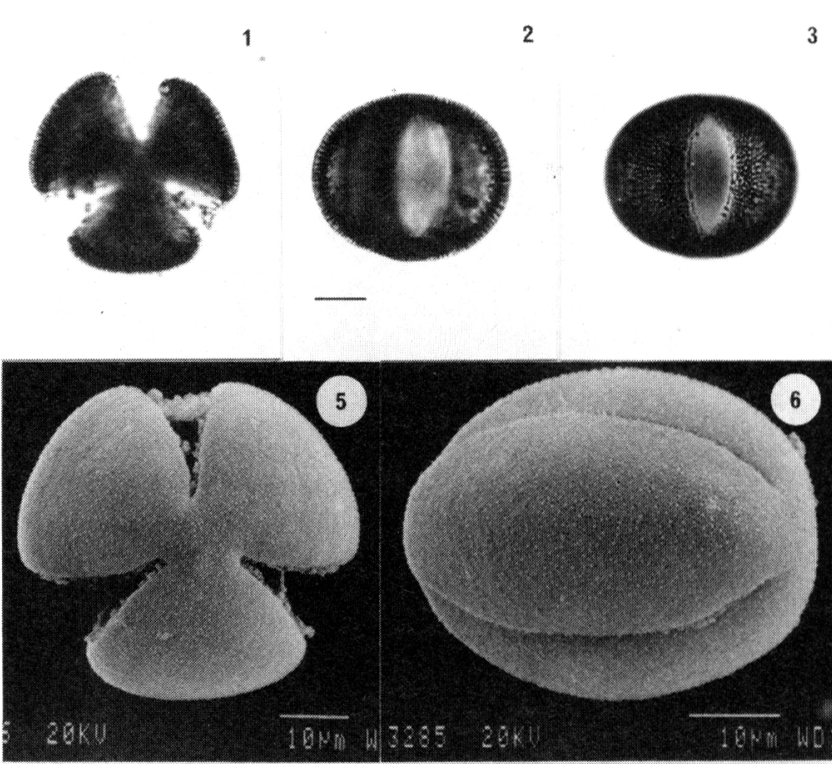

8
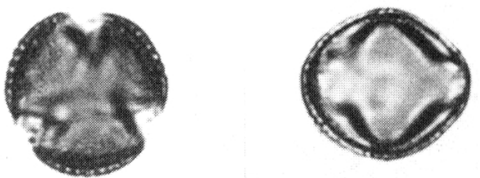

4
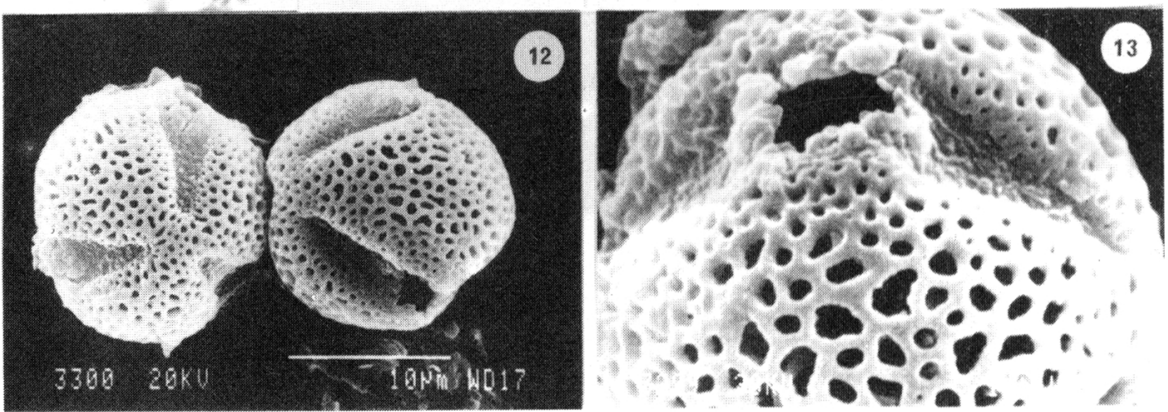

Lámina VII. Convolvulus tricolor: 1) v.p. y c.o.e. 2) v.e. y c.o.m. 3) v.e., superficie y abertura. 4) Detalle de la superficie y abertura. 5) Aspecto general del polen, v.p. 6) Aspecto general del polen, v.e. 7) Detalle de la estratificación de la exina. Wigandia caracasana: 8) v.p. y c.o.e. 9) v.e. y c.o.m. 10) v.e., costillas 11) v.e., superficie 12) Aspecto general del polen. 13). Detalle de la superficie y abertura. Escala M.O.: $10 \mu \mathrm{m}$. 